\title{
Multi-Point Mapping of Thunderstorm Electrical Structures
}

\author{
Cameron Fischer ${ }^{1}$, Cole Hanson ${ }^{1}$ \\ ${ }^{I}$ Carthage College, Department of Physics and Astronomy * \\ Kenosha, WI, United States of America
}

\begin{abstract}
Electric field measurements are necessary to understand thunderstorm evolution and lightning initiation. However, most existing measurements are made with single instruments carried by weather balloons. It is difficult to interpret such data since a change in observed electric field could be due either to motion of the instrument or charging/discharging currents. In order to decouple these behaviors, it is necessary to make simultaneous measurements at multiple locations. To avoid the complexity of multiple balloon launches, we describe a single balloon instrument with multiple, independent dropsondes to be released at desired time intervals. The dropsondes are designed to rotate and be self-stabilizing, enabling them to measure electric fields as they fall. The dropsondes are lightweight, robust, and low-cost, and include a preamplifier, GPS receiver, search coil and accelerometer for orientation sensing, microcontroller, and a telemetry system to transmit data to a ground station. Prototype instruments have been drop-tested to demonstrate aerodynamic stability and rotation and have been calibrated for electric field measurement. A balloon payload set to release a set of such dropsondes via hot-wire release mechanisms can thus accomplish the goal of multi-point measurements of thunderstorm electrical structures.
\end{abstract}

\section{Introduction}

Thunderstorms are complex thermodynamic systems that are constantly evolving. They evolve via several different mechanisms. The most important ones are the movement of mass on air currents, which leads to the movement of charge throughout the system due to changes carried on hydrometeors. In particular, the charging mechanisms are poorly understood, leading to a significant gap in our understanding of the charging, discharging, lightning initiation, and evolution that takes place inside the clouds. A typical storm structure anatomy shown to enthusiasts is depicted in Figure 1; however, this model lacks realism. Many assumptions are made in the model that we know to be not true. It has been known for a long time that storms get more charged over time, and lightning comes around to discharge them, yet we continue to rely on stagnant cartoon models to make predictions.

To better understand lightning and its effects, we must first fundamentally change our understanding of the charge structures present in storms. Current methods of measuring thunderstorm electric fields are limited because they cannot differentiate time evolution from spatial structure. This limitation is due to the method of measurement. Traditionally a single balloon-borne electric field sensor (Stolzenburg, 1999) is released to rise through a storm over the course of about half an hour and slowly record electric field vectors as it goes. Typical analysis of such data assumes that the storm's electric field does not change significantly over this timespan.

\footnotetext{
* This project would not have been possible without the generous Funding from Wisconsin Space Grant Consortium and the Birkeland Center for Space Science, and facilities offered through the Carthage College division of natural science Student Undergraduate Research Experience (SURE) program.
} 
This assumption is not particularly accurate, considering active thunderstorms can produce a lightning discharge every 10 seconds or so and can grow or dissipate significantly over the course of 20 minutes.

To overcome this limitation, we propose taking simultaneous measurements at multiple locations; this would allow us to separate time evolution from the spatial structure by providing a sort of snapshot of the storm's overall structure at varying points in time. Such multi-point measurements can be made efficiently by utilizing a single weather balloon to release multiple dropsondes into the storm. Our dropsondes are small self-contained measurement devices released from the top of the storm and measures electric fields as they fall. The first limitation this helps overcome is it shrinks the overall timespan of the measurements from the hour's scale to the minutes scale. Second, by releasing the dropsondes in intervals rather than all at once, we can give them some spatial and temporal separation between measurements. This allows us to obtain not only the overall charge structure as previous methods have done, but it also lets us see the charge structure stepping forward in time to see the evolution and movement of charge.

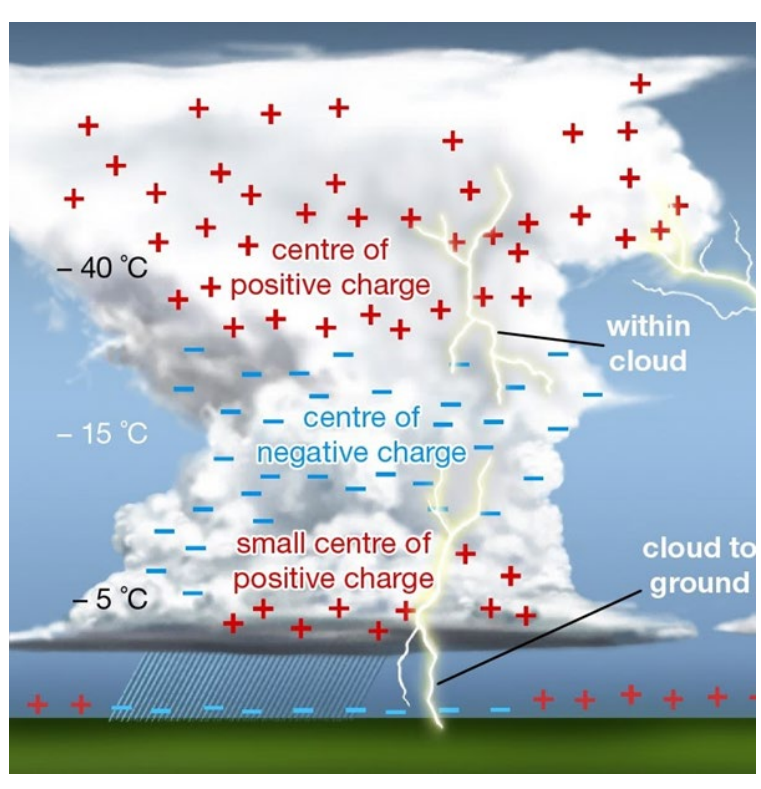

Figure 1

The above image (Krider, 2020) shows a cartoon-like depiction of thunderstorm cloud charge structures. A characteristic central negative region is sandwiched between an upper and lower positive region.

\section{Measurement Device}

\subsection{External design}

Each Dropsonde must function as a complete measurement suite. As such, the core elements of the design draw heavily on previous Thunderstorm electric field measurement devices, specifically the balloon-borne electric field meter. Its general principle relies on a rotating insulating tube with conductive electrodes attached to opposing sides. Charge is exchanged between the electrodes, providing a measurable quantity proportional to the electric field present. The dropsonde concept works on similar principles; Small electrodes on the dropsonde's body are used to accumulate charge inside of the storm's electric field. A spinning motion then forces the accumulated charge to flow between opposing electrodes, which can be measured to give a quantity proportional to the strength of the electric field. Finally, data is sent to the ground via an onboard telemetry system. 
A few design constraints must be met for this to work. Referring to figure 1, most electric fields ought to be vertically aligned, meaning the electrodes should therefore be vertically staggered and rotate about the horizontal axis. An Ellipsoid body shape provides the aerodynamic stability to keep the dropsondes rotational axis horizontal, as depicted in figure 2 . This property has been verified by simulation and prototype drop tests. Fins are designed directly into the body

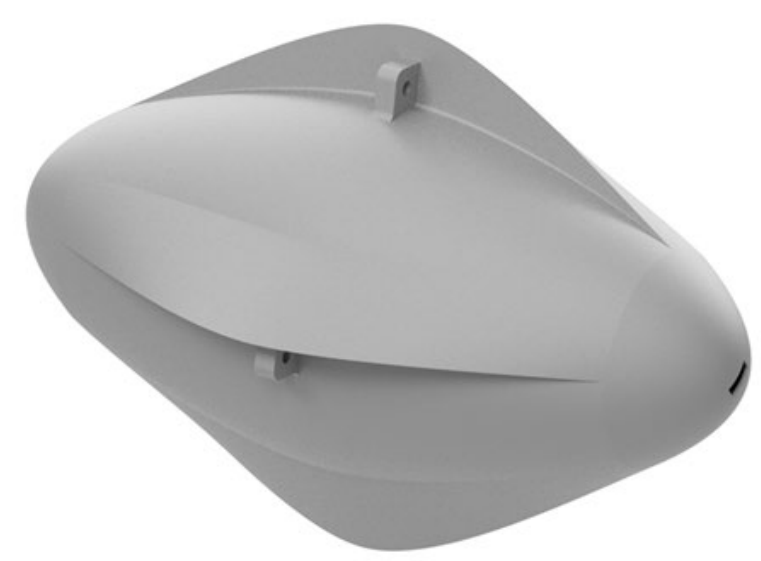

to cause rotation while falling, enabling the flow of charge between electrodes. Two sets of opposing faces allow two pairs of electrodes to be placed directly onto the body. This additional electrode pair allows for more confidence in measurements as each dropsonde has two independent measures of electric fields. Finally, the body itself acts as a shell to protect the internal electronics from water and debris usually found in storms.

\section{Figure 2}

The above figure shows the shell design for the dropsonde. The long elliptical body ensures a stable position while falling, and the fins generate a rotational force. The body is printed in 2 halves and secured together using lightweight nuts and bolts.

\subsection{Internal systems}

The four external electrodes are connected internally via an intermediate pre-amplifier, which intercepts the flow of charge, amplifies the signal to an appropriate level, and feeds that signal over to the central processing unit. Along with the pre-amp and CPU, several other peripherals inside the body help analyze the data and ensure the most accurate measurements. The first is a GPS, which allows each dropsonde to know its position in space and other helpful information like the current time and its velocity. An accelerometer also allows each dropsonde to reliably know its rate of rotation, which is later helpful in determining electric field strength. There is also a magnetic search coil, which is wrappings of magnet wire about the long face of the dropsonde. The coil interacts with the Earth's magnetic field as it rotates to produce a signal which, taken in conjunction with the electric field readings, will give a method of determining the direction the probe is facing. Finally, there is a telemetry broadcast system for transmitting data to a ground station rather than storing data onboard. The transmitter operates in the 915 $\mathrm{MHz}$ band. Figure 3 shows a block diagram of how each system is connected and interacts with one another. Each of these internal components is secured to a single interconnect PCB, which functions to connect all the devices and add rigidity to the body as a sort of internal brace. 
All the systems that originate from the pre-amp are analog. In contrast, the CPU is inherently a digital system, so there is some flexibility in how fast measurements are sampledutilizing a PJRC Teensy 4.0, the current system samples each analog peripheral at around 30,000 samples per second. Since there are two electrode pairs, an accelerometer, and a search coil connected, the CPU reads and processes about 120,000 12-bit numbers every second. The problem is that the telemetry system cannot broadcast data as fast as the CPU can read it. Due to transmitter bandwidth limits, noise considerations, and the need for error correcting codes, the telemetry system can only transmit around one thousand bits per second.

For this reason, the CPU compresses the raw data into packets containing simple statistics that represent the whole data set. For example, the CPU compresses the electrode signal from 30,000 individual numbers down to its mean value, minimum, maximum, variance, amplitude, phase, and frequency. It does a similar process for the accelerometer and search coil, and bundles in the location and time from GPS.

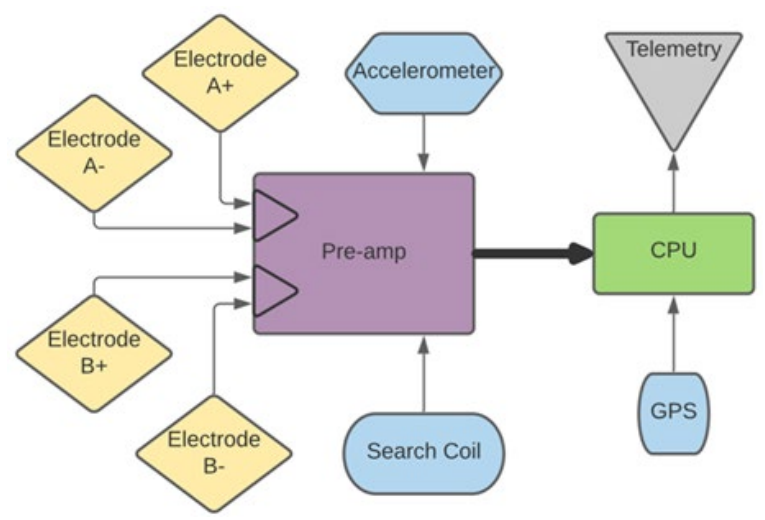

Figure 3

The figure above shows a block diagram of the internal systems and their relation to each other. The electrodes, accelerometer, and search coil are fed into the pre-amp, which relays information to the microcontroller (CPU) and finally receives GPS information and packages data for transmission via the telemetry system.

\section{Instrument Calibration}

Measuring the flow of charge across electrodes gives a value proportional to the electric field; therefore, it is necessary to calibrate the system to retrieve any sensible data back. To facilitate this calibration, a simple testing rig was manufactured to fulfill two main requirements; be able to hold onto the dropsonde in such a way that it can rotate freely about its long axis, but is constrained in all other directions, and be able to easily manipulate an electric field around the dropsonde. We produced the electric field by making two large conductive plates and attaching them to opposite charges of a power supply. Acting as a giant capacitor, the electric field between the plates can approximately be determined from the voltage between plates and the physical space separating them. To hold onto the dropsonde, a crude wooden rig was fabricated to support only the ends of the dropsonde allowing for minimal friction as it rotates. A leaf blower was then used to induce spin on the dropsonde utilizing the fins of the shell so that we did not directly interfere with the electric fields present, as well as to simulate the dropsonde in freefall. Finally, to retrieve 
data from the system, a modified version of the telemetry system was used to transmit both packets and a limited number of raw data points.

While full data transmission via the modified telemetry system is not possible, we were able to obtain enough data points for sufficient analysis. The graph in figure 4 titled Example Raw Data shows roughly $1 / 2$ a second worth of data collection during a particular test. The horizontal axis is a relative measure of time in datapoint index, and the vertical axis is in 12-bit ADC units. The clean sinusoidal shape of the curves, especially the electrode data, is a good indicator that the systems are working as intended. As was briefly discussed in section 2.2, the relative phase angle between these two functions and the functions' amplitudes can give some insight into the relative direction that the dropsonde is facing. This is important for understanding the path the dropsonde took as it fell as well as interpreting the non-vertical components of the electric field present in the storm clouds.

The graph in figure 4 titled Signal strength and Frequency shows the amplitude of electric field measurements over time, along with the rotational frequency of the dropsonde. The time scale is slightly longer here, showing roughly 30 seconds worth of condensed data packets. Both frequency and amplitude show very similar trends over time, an expected outcome. This helps to show that both amplitude and frequency data are needed to determine the true electric field strength. Previous work by a colleague, Max Becher, was done to derive this very relationship (Becher, 2019), which can be expressed as $|E|=\frac{A \tau \omega}{\sqrt{\tau^{2} \omega^{2}+1}}|V|+E_{0}$, where $\omega$ is the rotation velocity and $\mathrm{V}$ is the amplitude of the electrode signal. $\mathrm{A}, \tau$, and $E_{0}$ are constants to be set by calibration and will be determined using the final electronics and shell design.
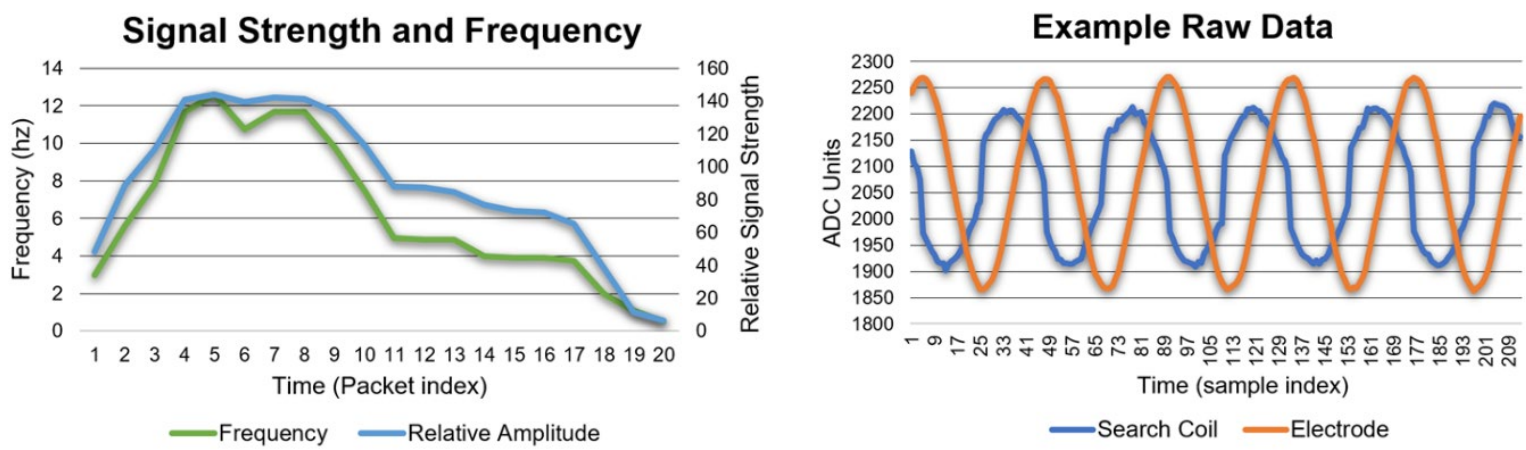

Figure 4

The two graphs above depict data output as transmitted by the dropsonde. The graph on the right shows individual data points from one of the two electrode channels and the magnetic search coil channel. The significant result here is the clean sinusoidal curves and the relation of phase angle. The graph on the left shows condensed data packets of electrode amplitude throughout a 30-second test run with varying rotation speeds also shown. The primary focus is the relationship between rotational frequency and electrode amplitude 


\section{The Mothership}

Most of the design work on the Mothership, the container which holds onto the dropsondes as they ascend to the top of thunderstorm clouds, was completed during a previous project in 2020 and was submitted to the WSGC proceedings under Multi-Point Mapping of Thunderstorm Electrical Structure, Evolution, and Resulting Phenomena (Meyer, 2020). In summary, the Mothership is designed to store six dropsondes during the balloon flight up about 15,000 meters, a typical height for thunderstorm clouds. The Mothership has the multifaceted purpose of being cheap but robust, holding onto the dropsondes, protecting them from wind and possible debris, and finally releasing the dropsondes at set intervals. The Mothership is made of almost entirely recycled materials to cut down on cost and weight, apart from the electronics that power the GPS and hot-wire cut-down mechanism.

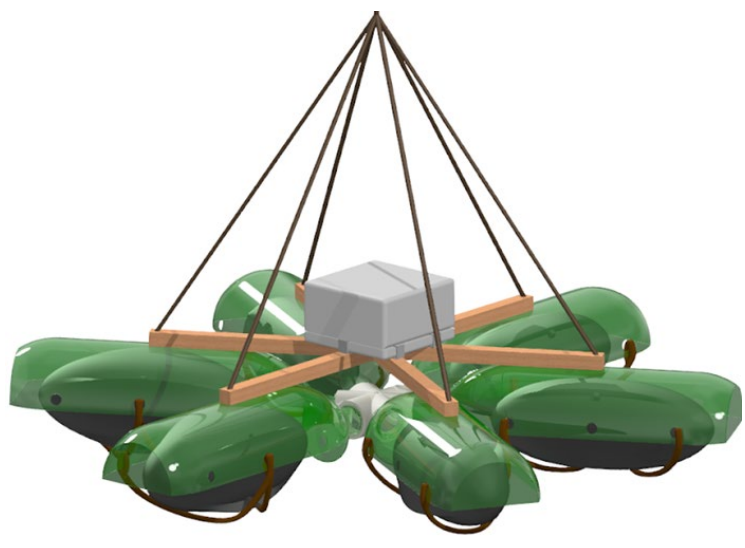

Figure 5

The above figure shows a rendition of the Mothership design, showing all six dropsondes and connections to a weather balloon not depicted (Fischer, 2020).

The Mothership has a GPS to determine when to release the dropsondes and if there is a problem in the flight path or timing. The hot wire cut-down system activates if all goes well in the journey and the Mothership makes it up to its desired altitude. The general principle is very simple: a thin wire will heat up if a large current is passed through it. This is the same process utilized in most household toaster ovens. Depending on how the dropsonde is secured, triggering the hot-wire mechanism can simply release the dropsondes or cause them to unwind and begin rotating as they are released. Several tests have been performed at a 100-meter altitude to ensure the repeatability of the hot wire cut-down system and from the limited number of tests done so far, the system works. More testing is still required to verify the repeatability thoroughly, but preliminary tests have shown very promising results.

\section{Conclusion and Future Work}

With the culmination of work done over the past year, this method of multi-point mapping of electric fields is more than just a good idea, it is a working system that simply requires more calibration work to get fully operational. More testing to prove the viability of the aerodynamics of the body is absolutely required, along with many more design iterations. From the few stability tests performed, there have already been signs of failure which require more work to correct. It will also be necessary to perform low temperature tests on the system. Temperatures in the upper levels of storms can reach as low as -45 Celsius, which can have very negative effects on battery usage. The final steps of this project are to perform full system high altitude tests and full system 
operation. After these high-altitude tests, simply analyzing the data and reaching new conclusions would be the goal, and something that is very within reach of this project. This work was presented at the 2021 American Geophysical Union Fall Meeting (Fischer, 2021) in conjunction with the work accomplished by William Schuster on the data processing of dropsondes (Schuster, 2021).

\section{Acknowledgments}

We would like to thank Dr. Brant Carlson for his guidance as an advisor as well as the Wisconsin Space Grant Consortium (WSGC), the Birkeland Center for Space Science, and the Carthage College division of natural science Student Undergraduate Research Experience (SURE) for funding, facilities, and equipment. The work presented above was done in conjunction with William Schuster's Development of Novel Data Analysis Techniques for Multi-Point Mapping of Thunderstorm Electrical Structures.

\section{References}

Becher, M. J. (2019) Calibration of a low-cost electric field sensing dropsonde for use in a thunderstorm sounding experiment, Carthage College Senior thesis

Becher, M. J. and Carlson, B. E. (2018), "Low-cost dropsonde development for multi-point measurement of thunderstorm electric fields," American Geophysical Union Fall Meeting 2018, Washington DC, USA, no. AE11B-2700, Dec. 2018.

Fischer, C., Hanson, C., Scheunemann, Z., Schuster, W., and Carlson, B. E. (2021) Dropsonde Design, Calibration, and Testing for Multi-Point Measurement of Thunderstorm Electrical Structures, American Geophysical Union Fall Meeting 2021, no. AE35A-1912, Dec. 2021

Fischer, C., Scheunemann, Z., Becher, M. J., and Carlson, B. E. (2020) Multi-point Measurement of Thunderstorm Electric Fields by Balloon-borne Dropsondes, American Geophysical Union Fall Meeting 2020, doi.org/10.1002/essoar.10505882.1, Dec. 2020

Krider, E. P. (n.d.). Thunderstorm electrification. In Encyclopcedia Britannica. Retrieved 2021, from https://www.britannica.com/science/thunderstorm/Thunderstorm-electrification. electrical charge distribution in a thunderstorm

Meyer, H., Schulds, G., Scheunemann, Z., Fischer, C., and Ellie, M. (2020). Multi-Point Mapping of Thunderstorm Electrical Structure, Evolution, and Resulting Phenomena, WSGC Proceedings submission

Schuster, W., Schulder, G., Fischer, C., Hanson, C., and Carlson, B. E. (2021), Development of Novel Data Analysis Techniques for Multi-Point Mapping of Thunderstorm Electrical Structures, American Geophysical Union Fall Meeting 2021, no. AE53A-1911, Dec. 2021

Stolzenburg, M. (1999). Atmospheric Physics Research. Modes of Research - Atmospheric Physics at UM. Retrieved 2021, from https://olemiss.edu/research/atmospheric physics/modes.html. balloon-borne electric field meter 